\title{
Always look at both sides of the heart: A double-orifice mitral valve discovered in a young adult with repaired tetralogy of Fallot
}

\author{
Ewa Kowalik, Anna Klisiewicz, Urszula Skrzypczyńska-Banasik, Piotr Hoffman
}

Department of Congenital Heart Diseases, Institute of Cardiology, Warsaw, Poland

An 18-year-old male with a history of tetralogy of Fallot repair when he was an infant was referred from the aforementioned pediatric center. He reported excellent exercise tolerance. On the physical examination no signs of heart failure were found. A transthoracic echocardiographic study showed preserved function of the enlarged right ventricle (Fig. 1A) and moderate pulmonary insufficiency (Fig. 1B). In addition, two-dimensional echocardiography in the parasternal short axis view (Fig. 1C) and three-dimensional (3D) transthoracic imaging (Fig. 1D) revealed that the mitral valve was divided with a fibrous bridge in two orifices of equal size (double-orifice mitral valve [DOMV]). Neither regurgitation nor obstruction of the valve was found (Fig. 1E, F). No other left-sided heart abnormalities were identified. The patient was submitted to routine clinical follow-up. The presentation of DOMV in adulthood is very rare. The anomaly might be isolated, but usually it coexists with endocardial cushion defects or left-side heart anomalies. DOMV associated with tetralogy of Fallot is an extremely unusual combination of congenital malformations. According to the classification proposed by Trowitzsch in 1985, in the presented case, the most commonly seen echocardiographic form of DOMV, i.e. complete bridge, was diagnosed. 3D echocardiography helps to assess mitral valve morphology and in the current case the transthoracic $3 \mathrm{D}$ imaging was sufficient to determine the type of malformation. The case stresses the importance of careful imaging in adult congenital heart disease patients even if transferred from paediatric centres. It is noteworthy to be surprised.

Conflict of interest: None declared

Address for correspondence: Ewa Kowalik, MD, PhD, Department of Congenital Heart Diseases, Institute of Cardiology, ul. Alpejska 42, 04-628 Warszawa, Poland, tel +48 2234344 00, fax +48 2234345 38, e-mail: ewa.kowalik@gmail.com 


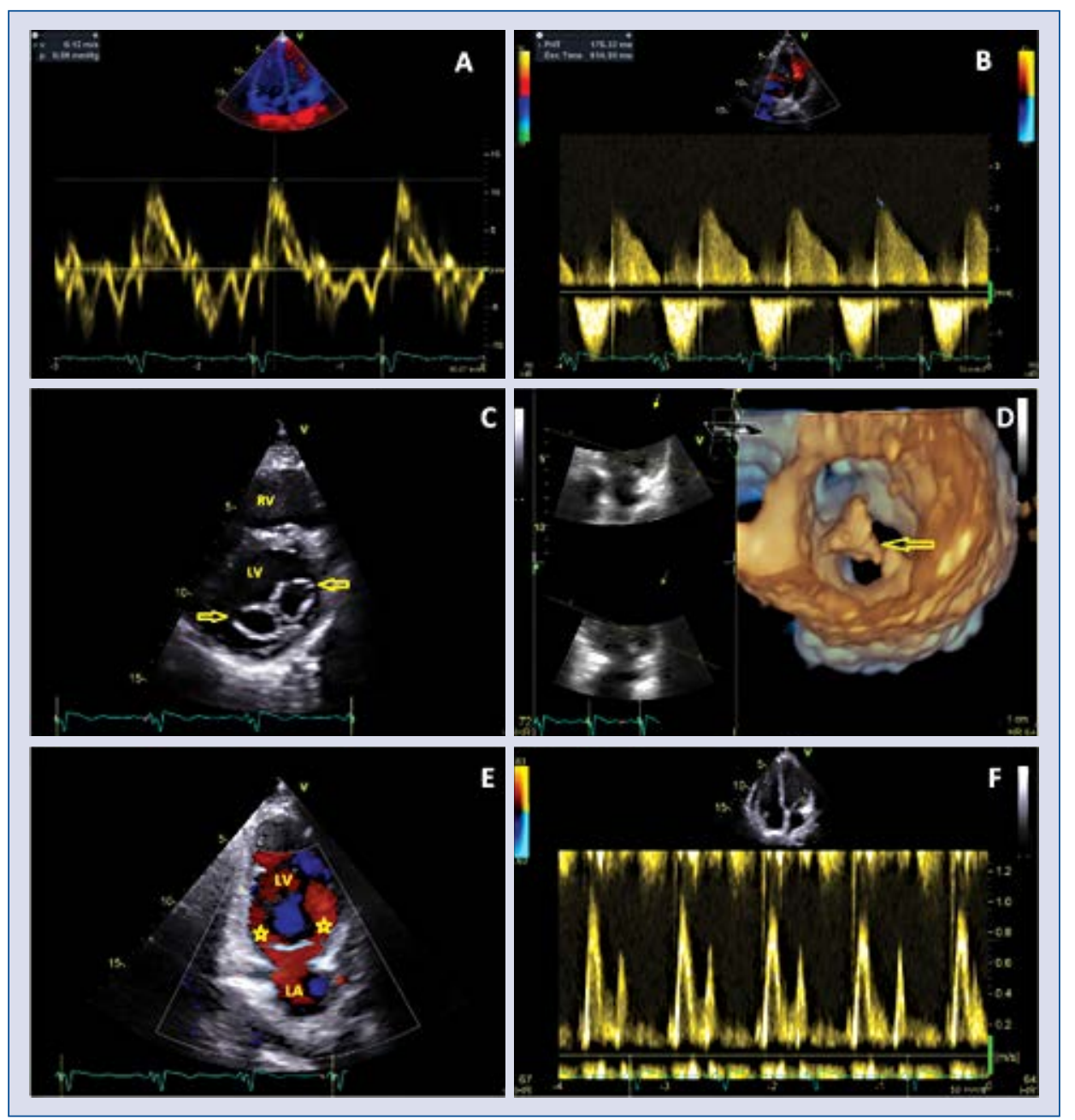

Figure. 1. Two-dimensional and three-dimensional (3D) echocardiographic study of a patient with repaired tetralogy of Fallot and newly diagnosed double-orifice mitral valve. Tissue Doppler imaging in the apical four-chamber view revealed preserved function of the enlarged right ventricle (A). In a parasternal short axis view a color Doppler guided continuous wave regurgitant pulmonary flow of moderate degree was also recorded (B). C. Parasternal short axis view in transthoracic echocardiography. Two separate mitral valve orifices of equal size were observed (arrows). D. Transthoracic 3D image of mitral valve. Mitral valve orifice divided by bridging structure clearly demonstrated at the left ventricular view (arrow). E. Color Doppler flow velocity mapping in an apical view demonstrated two separate jets of left ventricular filling (asterisks). Pulsed wave Doppler imaging confirmed unrestricted opening of the valve (F); LA — left atrium; LV — left ventricle; RV — right ventricle. 